\title{
Two-Dimensional Optical Imaging of Artificial Magnetic Field in the Laboratory
}

\author{
X.-N. Li, N. An, X.-J. Li*, X.-Q. Feng AND J.-T. BAI \\ Institute of Photonics and Photon-Technology, Northwest University, Xi'an 710069, China
}

(Received November 27, 2011; in final form October 14, 2012)

Two-dimensional images of the artificial magnetic field created by permanent magnets were obtained by means of the Faraday magnetic rotation effect. This provides a convenient and effective means to observe and measure macro-scale magnetic field, as well as lays a base for three-dimensional optical imaging of magnetic field. The magnetic field imaging here can also be considered as a key part of the magnetic rotation geomagnetic field imaging method which we put forward before, and will provide experimental support for this method.

DOI: 10.12693/APhysPolA.123.34

PACS: 42.25.Ja, 42.30.-d, 78.20.Ls

\section{Introduction}

We developed a new optical imaging measurement method of the geomagnetic field which we call magnetic rotation geomagnetic field imaging method [1]. The main idea is as follows: take the sun as the light source. With the help of the polarization characteristics of the sunlight reflected from the earth surface and the magnetic rotation effect of the atmosphere, measure the rotation angle of the polarized light at satellite after it passed through the atmosphere. Then the information of the geomagnetic field will be obtained. Finally, the distribution of the geomagnetic field will be resumed by some special ways.

In this paper, the Faraday magnetic rotation effect is applied for the two-dimensional optical imaging of the artificial magnetic field in the laboratory. This work can be considered not only as an independent research, but also a part of the magnetic rotation geomagnetic field imaging method.

The research on the image of magnetic field by means of the magneto-optic effect dates from the observation on magnetic domain of the ferromagnet [2, 3]. From then on, researchers from all over the world carried out a lot of experimental and theoretical researches on magnetic domain structures of different magnetic materials and their property with the help of magneto-optic effect. Recently, researches on the image of the magnetic domain are still very active $[4,5]$. Besides static domain images, researches also involved magnetic domain wall structure and dynamic variations of magnetic domain and magnetic domain wall and so on.

The researchers also developed the magneto-optic eddy current imaging technology [6]. The eddy current in conductor is produced by electromagnetic induction, and its magnetic field is detected by using the magneto-optic effect, then distribution of the eddy current will be ob-

\footnotetext{
*corresponding author; e-mail: lixiaojun@nwu.edu.cn
}

tained. Thus real-time image of the corrosion and fatigue crack in the mechanical part can be obtained. The technology has been widely used in the field of nondestructive test, especially for the important aircraft components. Research in this field is an international hot topic presently [7-9]. This technology plays an important role in the detection of the subsurface defect.

Both of magnetic domain imaging and magneto-optic eddy current imaging are applicable in micro-magnetic field. For macro-scale magnetic field (such as magnetic field of permanent magnet), iron dust was adopted to show magnetic line of force [10] in the early physics teaching and experiment. Later, the BELL company in the U.S. developed a kind of stereoscopic imaging system for permanent magnet magnetic field (MAGSCAN) based on the Hall probe scanning and computer plotting. Research on macro-scale magnetic field imaging using magneto-optic method has not been reported yet.

In the experiment of this paper, magnetic rotation of polarized light is measured by an imaging system to get two-dimensional image of the magnetic field. While the polarization analyzer is turning, light intensity on each pixel of the imaging plane will change synchronously. Collect a series of pictures of the light intensity distribution on imaging plane while the analyzer is turning, and process these pictures by computer program, then magnetic rotation angle of the light ray at any pixel will be found. The two-dimensional distribution of these rotated angles can represent two-dimensional image of the magnetic field.

By means of optical imaging of local magnetic field in the laboratory (such as the magnetic field of permanent magnet or electromagnetic devices), we can get distribution image of the magnetic field visually. This is important to many experiments and researches. The research in this paper will provide the magnetic field distribution images and measurement data for related physics experiments and studies, and will also provide experimental support for the magnetic rotation geomagnetic field imaging method. 


\section{Experimental device}

The magnetic field imaging here falls into two types: transmission and reflection.

The experimental device for the transmission imaging is shown in Fig. 1. The light source is a semiconductor laser, whose output is green light with wavelength of $532 \mathrm{~nm}$. (Blue light is supposed to be adopted in the magnetic rotation geomagnetic field imaging method [1]. However, limited by experimental conditions and funds, the green laser is used here.) Through the polarizer, the unpolarized light becomes vertically linearly polarized. After passing through the concave lens and convex lens 1 , it becomes a wide parallel beam with a diameter of about $100 \mathrm{~mm}$. This light beam vertically goes through a $10 \mathrm{~mm}$ thick ZF6 glass plate. Then it is converged by convex lens 2 and analyzed by the analyzer. Finally, it is received by CCD. The images collected by CCD are stored in computer for post-processing. Some permanent magnets are symmetrically fixed on the front and back surfaces of the ZF6 glass plate so as to provide magnetic field. Under this magnetic field, the polarized light will undergo a magnetic rotation while passing through the ZF6 glass plate. Here, the ZF6 glass plays a role of magnetic rotation medium.

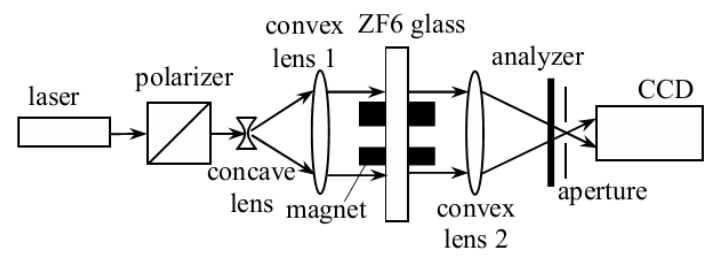

Fig. 1. Experimental device of the transmission imaging.

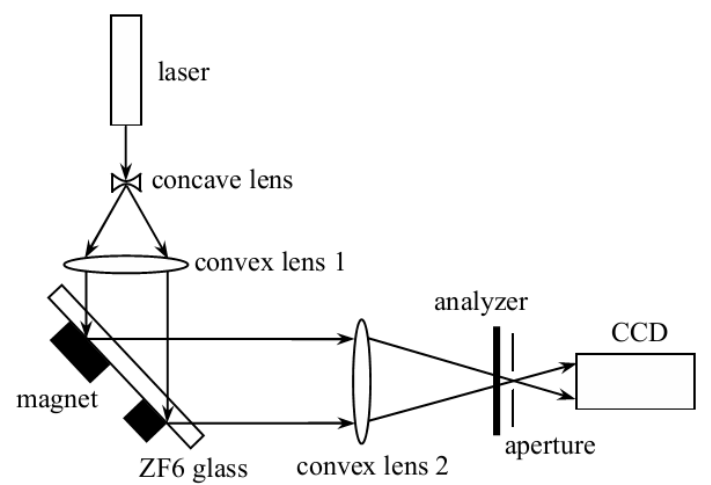

Fig. 2. Experimental device of the reflection imaging.

The experimental device of the reflection imaging is almost the same as that of the transmission imaging, except that the arrangement of the optical path is somewhat different. In Fig. 2, after passing through the concave lens and the convex lens 1 , the laser beam is expanded to a parallel beam with a diameter of about $100 \mathrm{~mm}$. This parallel beam is reflected at the back surface of the ZF6 glass plate and converged by convex lens 2. Then it goes through the analyzer and the aperture. Finally, it arrives at CCD. Here the permanent magnets to produce the magnetic field are installed on the back surface of the ZF6 glass plate.

The reflection imaging can be divided into two cases here. In one case, the incident light is linearly polarized. A polarizer should be installed between the laser and the concave lens in Fig. 2 to produce a vertical linearly polarized light. In another case, there is no polarizer in the optical path (as shown in Fig. 2). The natural light is directly reflected at the back surface of $\mathrm{ZF} 6$ glass plate and becomes linearly polarized or partially polarized. Magnetic rotation will take place while this reflected light is going through the glass plate. This somewhat simulates the whole process that the sunlight becomes polarized when it is reflected on the earth surface and then generates magnetic rotation in the geomagnetic field while it goes through the atmosphere in the magnetic rotation geomagnetic field imaging method [1].

\section{Image collecting}

Based on the experimental device in Fig. 1 and Fig. 2, while the analyzer is rotating, a series of pictures are taken by CCD and stored in the computer. Processing these pictures by image processing software, two-dimensional image of the magnetic field can be obtained.

In the experiment, the analyzer is a polaroid which is fixed in a vertically installed apertured universal stage. Accuracy of the universal stage is $0.01^{\circ}$. CCD camera is connected with the computer via an image collection card. We have developed an image collecting program whose user interface is shown in Fig. 3. Clicking the Save Bitmap button, the computer will drive the CCD camera to shoot grey level images. The images are stored in the computer in BMP format. Each image is composed of $768 \times 576$ pixels and each pixel holds 8 bits.

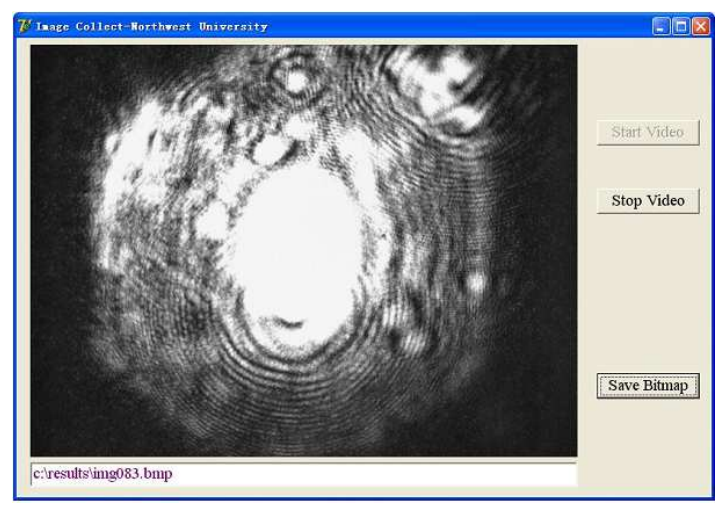

Fig. 3. Image collecting program.

When the analyzer is at zero, its transmission direction is horizontal. This zero angle corresponds to the extinction direction of the initial incident polarized light. While the analyzer is being turned continuously between 
the angle of $-\theta_{\max }$ and $+\theta_{\max }\left(\theta_{\max }\right.$ is determined by the magnetic field intensity), CCD collects images at intervals of a small angle $\Delta \theta$. These images are numbered from 0 to $N$ ( $N$ is an even number). The mid image numbered $N / 2$ is corresponding to the zero angle of the analyzer. No. 0 image is corresponding to $-\theta_{\max }$, and No. $N$ image to $\theta_{\max }$. Here $\theta_{\max }=\Delta \theta N / 2$, and the rotation angle of the analyzer of No. $k$ image can be calculated by the following formula:

$$
\theta_{k}=(k-N / 2) \Delta \theta \text {. }
$$

Two-dimensional imaging of the magnetic field of several shapes of permanent magnets and their different combinations are made. Herein courses and results of two experiments are given.

(1) Transmission imaging experiment

Two $14 \mathrm{~mm}$ long cylindrical permanent magnets with diameter of $5 \mathrm{~mm}$ are cling to the front and back surfaces of the ZF6 glass plate symmetrically as shown in Fig. 4. The effective imaging scope is within the broken circle in Fig. 4a. Rotation range of the analyzer is from $-5^{\circ}$ to $+5^{\circ}$. Images are shot at intervals of $0.05^{\circ}$. The images are named from img000 to img200, and the total number is 201 . The images are not all shown here limited by page space. To reflect the varying tendency of the images, we take one image every certain angle from zero angle (include zero angle) to both sides and enumerate them in Fig. 5.

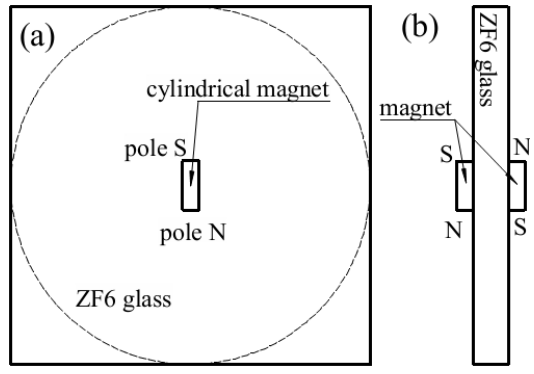

Fig. 4. Cylindrical magnet arrangement in the transmission imaging: (a) front view, (b) side view.

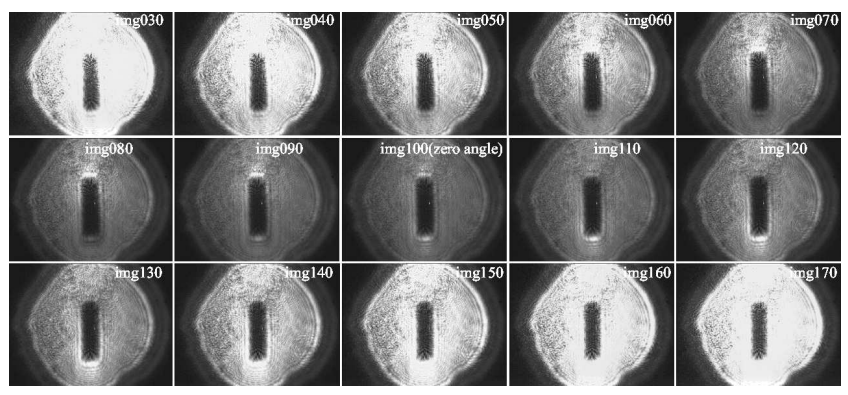

Fig. 5. Collected images of the transmission imaging.

(2) Reflection imaging experiment

Two disk-shaped permanent magnets (the magnetic poles are on the two faces of the disk) with thickness around $4 \mathrm{~mm}$ and diameter about $25 \mathrm{~mm}$, adhere to the back surface of ZF6 glass plate as shown in Fig. 6. The effective imaging scope is within the broken circle. Rotation range of the analyzer is between $-11^{\circ}$ and $+11^{\circ}$. Images are shot at intervals of $0.1^{\circ}$. The images are named from img000 to img220, and the total number is 221 . The collected images are selectively shown in Fig. 7.

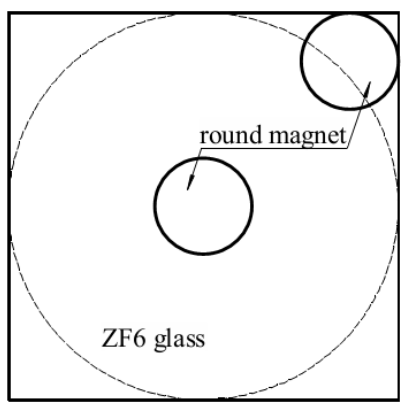

Fig. 6. Round magnet arrangement in the reflection imaging.

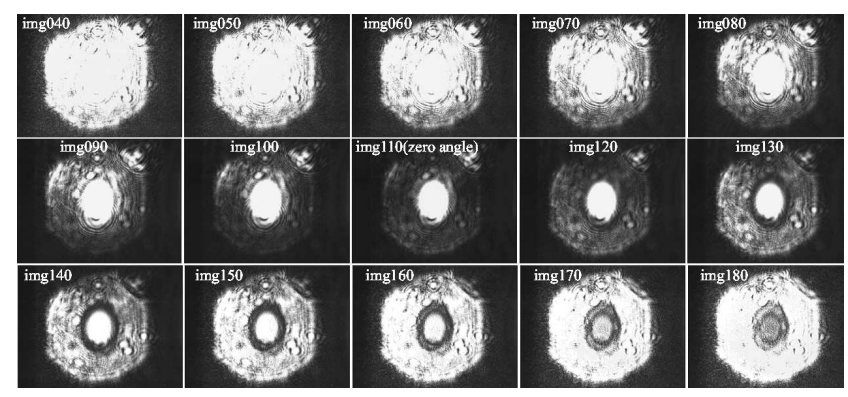

Fig. 7. Collected images of the reflection imaging.

\section{Image processing}

The images taken by CCD are not the actual image of the magnetic field. Only after the images have been processed, the final two-dimensional distribution image of the magnetic field can be obtained. According to the Faraday magnetic rotation effect formula [11],

$$
\theta=V B L,
$$

in a certain imaging course, the Verdet constant $V$ and the traveling length $L$ of the light in the medium are both unchanging, thus the rotation angle $\theta$ of the polarization plane is directly proportional to the magnetic field intensity. The image processing is aimed at getting the magnetic rotation angle of the polarization plane at every pixel so as to obtain the two-dimensional distribution of magnetic field within the imaging scope finally.

Actually, the image processing is a course of polarization analyzing. No. $k$ image is corresponding to a certain rotation angle $\theta_{k}$ of the analyzer. Scan the images and obtain grey level of every pixel. For a certain pixel $p_{i j}$, a curve that the grey level (corresponding to the intensity 
of the transmission light) varies with the rotation angle of the analyzer can be obtained. The rotation angle corresponding to the minimum grey level in this curve is the magnetic rotation angle for this pixel. This means that if

$$
H_{i j}^{k}=\min \left(H_{i j}^{1}, H_{i j}^{2}, \ldots, H_{i j}^{N}\right),
$$

where $H_{i j}^{k}$ is the grey level of pixel $p_{i j}$ in No. $k$ image, then

$$
\theta_{i j}=\theta_{k},
$$

where $\theta_{i j}$ is the magnetic rotation angle of the polarized light at pixel $p_{i j}$. This way of comparing the images, finding the minimum grey level, and ascertaining the polarization direction is light extinction method actually.

According to formula (2), the magnetic rotation angle of the polarization plane is directly proportional to the magnetic field intensity. Thus here the two-dimensional distribution of the magnetic rotation angle $\theta_{i j}$ is in the same pattern as the two-dimensional distribution of the magnetic field.

For a convenient view of two-dimensional distribution, the magnetic rotation angle $\theta_{i j}$ is shown in the form of grey level in a new image. This new image may be regarded as the magnetic field distribution image, which is stored and calculated in 8-bit data, i.e. it can describe grey level of 256 . When the computer program processes the images, the maximum rotation angle is represented by the saturated grey level 255, and the zero rotation angle by grey level 0 . The grey level is linearly related to the rotation angle. This relationship can be expressed as follows:

$$
H_{i j}=\left(\theta_{i j} / \theta_{\max }\right) 255 \text {, }
$$

where $H_{i j}$ is the grey level of pixel $p_{i j}$. A two-dimensional image based on the grey level $H_{i j}$ is the distribution image of the magnetic field. Via the formula (5) and (2), corresponding relationship between the grey level and the magnetic field intensity can be established.

According to processing method mentioned above, we built an image processing software which is given in Fig. 8.

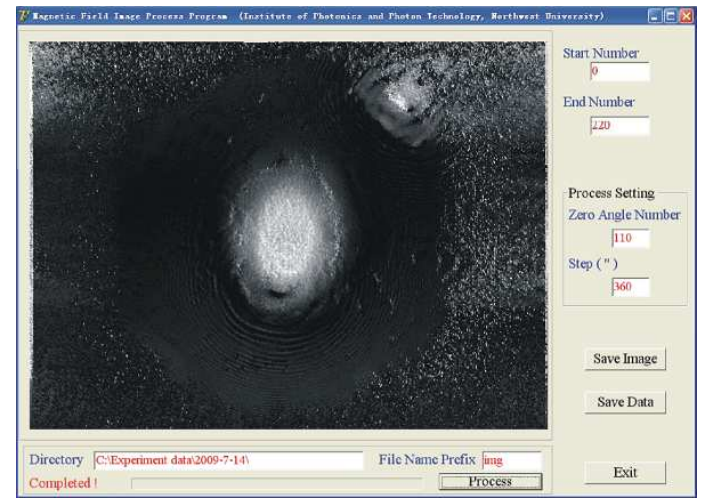

Fig. 8. Image processing program.

The processing results of the images:
(1) Transmission imaging

After the 201 images represented by images in Fig. 5 were processed, magnetic field distribution image of the small permanent magnets in Fig. 4 is obtained. The processing result is shown in Fig. 9. The magnetic field distribution nearby the two magnetic poles of the cylindrical magnet can be observed. Because the end faces (magnetic poles) of the cylindrical magnets are vertical to the glass surface, the magnetic field seems weak.

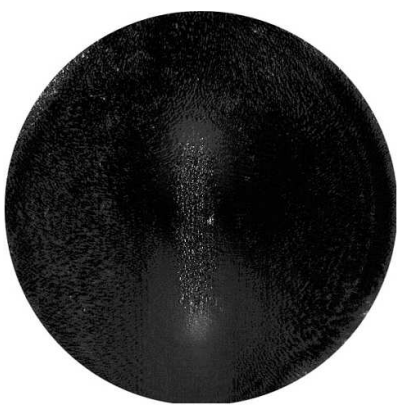

Fig. 9. Processing results of the transmission imaging.

(2) Reflection imaging

After the 221 images represented by images in Fig. 7 were processed, magnetic field distribution image of the round permanent magnet in Fig. 6 is obtained. The processing results are shown in Fig. 10. Strong magnetic field distribution of the magnetic pole of the round magnet is shown. The round magnet on the right upper corner is partially in the imaging scope and its magnetic field is also displayed.

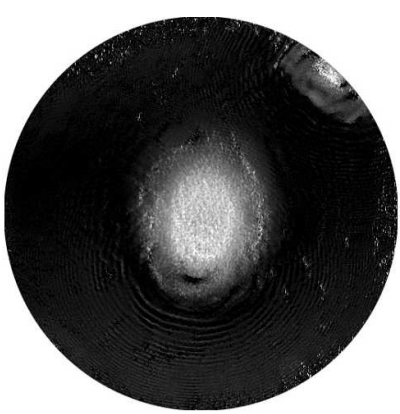

Fig. 10. Processing results of the reflection imaging.

\section{Summary}

Magneto-optic two-dimensional imaging cannot only build visual image, but also provide measurement data. It is a basis for magneto-optic three-dimensional imaging in our following research, too. Here, the measurement data of the magneto-optic two-dimensional imaging is actually average value of the magnetic field along the light path within the medium. If the medium is very thin, this average value can represent the magnetic field intensity of the test points. If the medium thickness is 
large and the traveling distance of the light through the medium is long, the magnetic field intensity of each point cannot be accurately reflected by the measurement data of magneto-optic two-dimensional imaging. In this case, on the basis of many two-dimensional imaging, three-dimensional imaging of magnetic field should be carried out by means of computerized tomography to restore the magnetic field intensity of each point.

In addition, the polarization analyzing method used here is the light extinction method whose precision is not high. On the basis of the researches in this paper, we design a new experimental project that combines the magneto-optic modulation and two-dimensional imaging. This design takes advantage of high polarization analyzing ability of the magneto-optic modulation, and is expected to remarkably improve precision of imaging and measurement.

As to the research of the magnetic field three-dimensional optical imaging and the magneto-optic modulation two-dimensional imaging, we will introduce them in forthcoming papers.

\section{Acknowledgments}

Supported by Research Project (No: 08JZ68, 2010JS111, 12JK0519) of Education Department of Shaanxi Province, China.

\section{References}

[1] X.J. Li, J.T. Bai, Y.A. Li, X.N. Li, Y.Y. Wang, X.Q. Feng, Prog. Nat. Sci. 18, 21 (2008).

[2] H.J. Williams, F.G. Foster, E.A. Wood, Phys. Rev 82, 119 (1951)

[3] J.F. Dillon, J. Appl. Phys. 29, 539 (1958).

[4] H. Ferraria, V. Bekerisa, T.H. Johansen, Physica $B$ 398, 476 (2007)

[5] Y. Ge, O. Heczko, O. Söderberg, S.-P. Hannula, Mater. Sci. Eng. A 481-482, 302 (2008)

[6] S. Simms, Mater. Eval. 50, 529 (1993).

[7] P. Ramuhalli, J. Slade, U. Park, L. Xuan, L. Udpa, Proc. SPIE 5062, 248 (2003)

[8] Y.H. Cheng, Z.F. Zhou, G.Y. Tian, Nondestructive Testing Evaluation Int. 40, 374 (2007)

[9] Y.H. Cheng, Z.F. Zhou, B.B. Yin, Opt. Precis. Eng. 14, 797 (2006) (in Chinese).

[10] Z.K. Meng, Exp. Teach. Instrum. 7-8, 61 (1999) (in Chinese).

[11] Y.J. Pan, J. Zou, Optoelectronic Technology, Chongqing University Press, Chongqing 2000 (in Chinese). 